\title{
SUPER-RIGID DONALDSON-THOMAS INVARIANTS
}

\author{
Kai Behrend And Jim Bryan
}

\begin{abstract}
We solve the part of the Donaldson-Thomas theory of Calabi-Yau threefolds which comes from super-rigid rational curves. As an application, we prove a version of the conjectural Gromov-Witten/Donaldson-Thomas correspondence of [MNOP] for contributions from super-rigid rational curves. In particular, we prove the full GW/DT correspondence for the quintic threefold in degrees one and two.
\end{abstract}

\section{Introduction}

Let $Y$ be a smooth complex projective Calabi-Yau threefold. Let $I_{n}(Y, \beta)$ be the moduli space of ideal sheaves $I_{Z} \subset \mathcal{O}_{Y}$, where the associated subscheme $Z$ has maximal dimension equal to one, the holomorphic Euler characteristic $\chi\left(\mathcal{O}_{Z}\right)$ is equal to $n$, and the associated 1-cycle has class $\beta \in H_{2}(Y)$.

Recall that $I_{n}(Y, \beta)$ has a natural symmetric obstruction theory [Th00], [BF05]. Hence we have the (degree zero) virtual fundamental class of $I_{n}(Y, \beta)$, whose degree $N_{n}(Y, \beta) \in \mathbb{Z}$ is the associated Donaldson-Thomas invariant.

Let

$$
C=\sum_{i=1}^{s} d_{i} C_{i}
$$

be an effective cycle on $Y$, and assume that the $C_{i}$ are pairwise disjoint, smoothly embedded rational curves with normal bundle $N_{C_{i} / Y} \cong \mathcal{O}(-1) \oplus \mathcal{O}(-1)$. Such curves are called super-rigid rational curves in $Y$ [Pa99, BP01]. Assume that the class of $C$ is $\beta$.

Let $J_{n}(Y, C) \subset I_{n}(Y, \beta)$ be the locus corresponding to subschemes $Z \subset Y$ whose associated cycle under the Hilbert-Chow morphism is equal to $C$ (see Definition 2.1). Since $J_{n}(Y, C) \subset I_{n}(Y, \beta)$ is open and closed (see Remark 2.2), we get an induced virtual fundamental class on $J_{n}(X, C)$ by restriction. We call

$$
N_{n}(Y, C)=\operatorname{deg}\left[J_{n}(Y, C)\right]^{\text {vir }}
$$

the contribution of $C$ to the Donaldson-Thomas invariant $N_{n}(Y, \beta)$.

The goal of this paper is to compute the invariants $N_{n}(Y, C)$.

To formulate our results, we define a series $P_{d}(q) \in \mathbb{Z}[[q]]$, for all integers $d \geq 0$ by

$$
\prod_{m=1}^{\infty}\left(1+q^{m} v\right)^{m}=\sum_{d=0}^{\infty} P_{d}(q) v^{d} .
$$

Received by the editors July, 182006. 
Moreover, recall the McMahon function

$$
M(q)=\prod_{m=1}^{\infty} \frac{1}{\left(1-q^{m}\right)^{m}} .
$$

Then we prove (Theorem 2.14) that

$$
\sum_{n=0}^{\infty} N_{n}(Y, C) q^{n}=M(-q)^{\chi(Y)} \prod_{i=1}^{s}(-1)^{d_{i}} P_{d_{i}}(-q) .
$$

Maulik, Nekrasov, Okounkov, and Pandharipande have conjectured a beautiful correspondence between Gromov-Witten theory and Donaldson-Thomas theory which we call the GW/DT correspondence.

As an application of the above formula we prove the GW/DT correspondence for the contributions from super-rigid rational curves (Theorem 3.1). In particular, we prove the full degree $\beta \mathrm{GW} / \mathrm{DT}$ correspondence (Conjecture 3 of [MNOP]) for any $\beta$ for which it is known that all cycle representatives are supported on superrigid rational curves (Corollary 3.2). For example, our results yield the GW/DT correspondence for the quintic threefold in degrees one and two (Corollary 3.3). As far as we know, these are the first instances of the GW/DT conjecture to be proved for compact Calabi-Yau threefolds.

The local GW/DT correspondence for super-rigid rational curves follows from the results of $[\mathrm{MNOP}]$ as a special case of the correspondence for toric Calabi-Yau threefolds. In contrast to Gromov-Witten theory, passing from the local invariants of super-rigid curves to global invariants is non-trivial in Donaldson-Thomas theory, and can be regarded as the main contribution of this paper.

1.1. Weighted Euler characteristics. Our main tool will be the weighted Euler characteristics introduced in [Be05]. Every scheme $X$ has a canonical $\mathbb{Z}$-valued constructible function $\nu_{X}$ on it. The weighted Euler characteristic $\widetilde{\chi}(X)$ of $X$ is defined as

$$
\tilde{\chi}(X)=\chi\left(X, \nu_{X}\right)=\sum_{n \in \mathbb{Z}} n \chi\left(\nu_{X}^{-1}(n)\right) .
$$

More generally, we use relative weighted Euler characteristics $\widetilde{\chi}(Z, X)$ defined as

$$
\widetilde{\chi}(Z, X)=\chi\left(Z, f^{*} \nu_{X}\right),
$$

for any morphism $f: Z \rightarrow X$. Three fundamental properties are

(i) if $X \rightarrow Y$ is étale, then $\widetilde{\chi}(Z, X)=\widetilde{\chi}(Z, Y)$,

(ii) if $Z=Z_{1} \sqcup Z_{2}$ is a disjoint union, $\widetilde{\chi}(Z, X)=\widetilde{\chi}\left(Z_{1}, X\right)+\widetilde{\chi}\left(Z_{2}, X\right)$,

(iii) $\tilde{\chi}\left(Z_{1}, X_{1}\right) \tilde{\chi}\left(Z_{2}, X_{2}\right)=\tilde{\chi}\left(Z_{1} \times Z_{2}, X_{1} \times X_{2}\right)$.

The main result of [Be05], Theorem 4.18, asserts that if $X$ is a projective scheme with a symmetric obstruction theory on it, then

$$
\operatorname{deg}[X]^{\mathrm{vir}}=\tilde{\chi}(X) .
$$

Thus we can calculate $N_{n}(Y, C)$ as $\tilde{\chi}\left(J_{n}(Y, C)\right)$.

We will also need the following fact. If $X$ is an affine scheme with an action of an algebraic torus $T$ and an isolated fixed point $p \in X$, and $X$ admits a symmetric obstruction theory compatible with the $T$-action, then

$$
\nu_{X}(p)=(-1)^{\operatorname{dim} T_{p} X},
$$


where $T_{p} X$ is the Zariski tangent space of $X$ at $p$. This is the main technical result of [BF05], Theorem 3.4.

Finally, we will use the following result from [BF05]. If $X$ is a smooth threefold (not necessarily proper), then

$$
\sum_{m=0}^{\infty} \widetilde{\chi}\left(\operatorname{Hilb}^{m} X\right) q^{m}=M(-q)^{\chi(X)} .
$$

In the case where $X$ is projective and Calabi-Yau, the above proves Conjecture 1 of $[\mathrm{MNOP}]$. Note that Conjecture 1 of [MNOP] has also been proved in [Li06] and [LP06].

\section{The Calculation}

2.1. The open subscheme $J_{n}(Y, C)$.

Definition 2.1. Let $C_{1}, \ldots, C_{s}$ be pairwise distinct, super-rigid rational curves on $Y$ and let $\left(d_{1}, \ldots, d_{s}\right)$ be an $s$-tuple of non-negative integers. Let $C=\sum_{i} d_{i} C_{i}$ be the associated 1-cycle on $Y$ and let $\beta$ be the class of $C$ in homology. Define

$$
J_{n}(Y, C) \subset I_{n}(Y, \beta)
$$

to be the open and closed subscheme consisting of subschemes $Z \subset Y$ whose associated 1-cycle is equal to $C$.

Remark 2.2. To see that $J_{n}(Y, C)$ is, indeed, open and closed, consider the HilbertChow morphism, see [Ko96], Chapter I, Theorem 6.3, which is a morphism

$$
f: I_{n}(Y, \beta)^{s n} \longrightarrow \operatorname{Chow}(Y, d),
$$

where $\operatorname{Chow}(Y, d)$ is the Chow scheme of 1-dimensional cycles of degree $d=\operatorname{deg} \beta$ on $Y$. It is a projective scheme. Moreover, $I_{n}(Y, \beta)^{s n}$ is the semi-normalization of $I_{n}(Y, \beta)$. The structure morphism $I_{n}(Y, \beta)^{s n} \rightarrow I_{n}(Y, \beta)$ is a homeomorphism of underlying Zariski topological spaces. Therefore the Hilbert-Chow morphism descends to a continuous map of Zariski topological spaces

$$
|f|:\left|I_{n}(Y, \beta)\right| \longrightarrow|\operatorname{Chow}(Y, d)| .
$$

Because the $C_{i}$ are super-rigid, the cycle $C$ corresponds to an isolated point of $\mid$ Chow $(Y, d) \mid$. So the preimage of this point under $|f|$ is open and closed in $\left|I_{n}(Y, \beta)\right|$. The open subscheme of $I_{n}(Y, \beta)$ defined by this open subset is $J_{n}(Y, C)$.

Definition 2.3. As $J_{n}(Y, C)$ is open in $I_{n}(Y, \beta)$, it has an induced (symmetric) obstruction theory and hence a virtual fundamental class of degree zero. Since $J_{n}(Y, C)$ is closed in $I_{n}(Y, \beta)$ it is projective, and so we can consider the degree of the virtual fundamental class

$$
N_{n}(Y, C)=\operatorname{deg}\left[J_{n}(Y, C)\right]^{\mathrm{vir}},
$$

and call it the contribution of $C$ to the Donaldson-Thomas invariant $N_{n}(Y, \beta)$. 
2.2. The closed subset $\widetilde{J}_{n}(Y, C)$.

Definition 2.4. Let $C=\sum_{i} d_{i} C_{i}$ be as above and denote by supp $C$ the reduced closed subscheme of $Y$ underlying $C$. Let

$$
\widetilde{J}_{n}(Y, C) \subset J_{n}(Y, C) \subset I_{n}(Y, \beta)
$$

be the closed subset consisting of subschemes $Z \subset Y$ whose underlying closed subset $Z^{\text {red }} \subset Y$ is contained in $\operatorname{supp} C$.

Remark 2.5. To see that $\widetilde{J}_{n}(Y, C)$ is closed in $I_{n}(Y, \beta)$, let $W_{m} \subset Y$ be the $m$-th infinitesimal neighborhood of $\operatorname{supp} C \subset Y$. For fixed numerical invariants $n$ and $\beta$ there exists a sufficiently large $m$, such that for any subscheme $Z \subset Y$, with invariants $n$ and $\beta$, and such that $Z^{\text {red }} \subset \operatorname{supp} C$, we have $Z \subset W_{m}$. For such an $m$, consider the Hilbert scheme $I_{n}\left(W_{m}, \beta\right)$, which is a closed subscheme of $I_{n}(Y, \beta)$, as $W_{m}$ is a closed subscheme of $Y$. The underlying closed subset of $I_{n}(Y, \beta)$ is equal to $\widetilde{J}_{n}(Y, C)$.

Remark 2.6. Informally speaking, $J_{n}(Y, C)$ parameterizes subschemes whose one dimensional components are confined to $C$, but may have embedded points anywhere in $Y$, whereas $\widetilde{J}_{n}(Y, C)$ parameterizes subschemes where both the one dimensional components and the embedded points are supported on $C$.

2.3. The open Calabi Yau $N=\mathcal{O}(-1) \oplus \mathcal{O}(-1)$. We consider the open CalabiYau $N$, which is the total space of the vector bundle $\mathcal{O}(-1) \oplus \mathcal{O}(-1)$ on $\mathbb{P}^{1}$. We denote by $C_{0} \subset N$ the zero section. We consider the Hilbert scheme $I_{n}\left(N,\left[d C_{0}\right]\right)$.

Let $\bar{N}$ denote $\mathbb{P}(\mathcal{O} \oplus \mathcal{O}(-1) \oplus \mathcal{O}(-1))$ and let $D_{\infty}=\bar{N} \backslash N$. Since $3 D_{\infty}$ is an anticanonical divisor of $\bar{N}$, the corresponding section defines a trivialization of $K_{N} . \bar{N}$ is naturally a toric variety, $D_{\infty}$ is an invariant divisor, and we let $T_{0}$ be the subtorus whose elements act trivially on $K_{N}$. Then $T_{0}$ induces a $T_{0}$-equivariant symmetric obstruction theory on $I_{n}\left(N,\left[d C_{0}\right]\right)$, by Proposition 2.4 of [BF05]. Moreover, the $T_{0}$ fixed points in $I_{n}\left(N,\left[d C_{0}\right]\right)$ are isolated points whose Zariski tangent spaces have no trivial factors as $T_{0}$ representations (the proof of Lemma 4.1, Part (a) and (b) in [BF05] is easily adapted to prove this).

As in [MNOP], the $T_{0}$ fixed points in $I_{n}\left(N,\left[d C_{0}\right]\right)$ correspond to subschemes which are given by monomial ideals on the restriction to the two affine charts of $N$. The number of such fixed points is given by $p(n, d)$ described below.

Let $p(n, d)$ be the number of triples $\left(\pi_{0}, \lambda, \pi_{\infty}\right)$, where $\pi_{0}$ and $\pi_{\infty}$ are 3 -dimensional partitions and $\lambda$ a 2-dimensional partition. The 3-dimensional partitions each have one infinite leg with asymptotics $\lambda$, and no other infinite legs. Moreover, $d=|\lambda|$ and $n$ is given by ([MNOP] Lemma 5$)$

$$
n=\left|\pi_{0}\right|+\left|\pi_{\infty}\right|+\sum_{(i, j) \in \lambda}(i+j+1),
$$

where the size of a three dimensional partition with an infinite leg of shape $\lambda$ along the $z$ axis is defined by

$$
|\pi|=\#\left\{(i, j, k) \in \mathbb{Z}_{\geq 0}^{3}: \quad(i, j, k) \in \pi,(i, j) \notin \lambda\right\} .
$$

Proposition 2.7. We have

$$
\tilde{\chi}\left(I_{n}\left(N,\left[d C_{0}\right]\right)\right)=(-1)^{n-d} p(n, d) .
$$


Proof. By Corollary 3.5 of [BF05], we have

$$
\tilde{\chi}\left(I_{n}\left(N,\left[d C_{0}\right]\right)\right)=\sum_{p}(-1)^{\operatorname{dim} T_{p}},
$$

where the sum is over all $T_{0}$-fixed points on $I_{n}\left(N,\left[d C_{0}\right]\right)$ and $T_{p}$ is the Zariski tangent space of $I_{n}\left(N,\left[d C_{0}\right]\right)$ at $p$. The parity of $\operatorname{dim} T_{p}$ can be easily deduced from Theorem 2 of [MNOP] (just as in the proof of Lemma 4.1 (c) in [BF05]). The result is $n-d$. So all we have to notice is that $p(n, d)$ is the number of fixed points of $T_{0}$ on $I_{n}\left(N,\left[d C_{0}\right]\right)$.

Corollary 2.8. We have

$$
\widetilde{\chi}\left(\widetilde{J}_{n}\left(N, d C_{0}\right), I_{n}\left(N,\left[d C_{0}\right]\right)\right)=(-1)^{n-d} p(n, d) .
$$

Proof. We just have to notice that all $T_{0}$-fixed points are contained in $\widetilde{J}_{n}\left(N, d C_{0}\right)$.

2.4. The box counting function $p(n, d)$. Counting three dimensional partitions with given asymptotics has been shown by Okounkov, Reshetikhin, and Vafa [ORV] to be equivalent to the topological vertex formalism which occurs in Gromov-Witten theory. They give general formulas for the associated generating functions in terms of $q$ values of Schur functions which we will use to prove the following Lemma.

Lemma 2.9. The generating function for $p(n, d)$ is given by

$$
\sum_{n=0}^{\infty} p(n, d) q^{n}=M(q)^{2} P_{d}(q),
$$

where the power series $P_{d}(q)$ and $M(q)$ are defined in Equations (1) and (2).

Proof. The generating function for the number of 3-dimensional partitions with one infinite leg of shape $\lambda$ is given by equation 3.21 in [ORV]:

$$
\sum_{\substack{\text { 3d partitions } \pi \\
\text { asymptotic to } \lambda}} q^{|\pi|}=M(q) q^{-\left(\begin{array}{l}
\lambda \\
2
\end{array}\right)-\frac{|\lambda|}{2}} s_{\lambda^{t}}\left(q^{1 / 2}, q^{3 / 2}, q^{5 / 2}, \ldots\right)
$$

where $\lambda^{t}$ is the transpose partition, $\left(\begin{array}{c}\lambda \\ 2\end{array}\right)=\sum_{i}\left(\begin{array}{c}\lambda_{i} \\ 2\end{array}\right),|\lambda|=\sum_{i} \lambda_{i}$, and

$$
s_{\lambda^{t}}\left(q^{1 / 2}, q^{3 / 2}, q^{5 / 2}, \ldots\right)
$$

is the Schur function associated to $\lambda^{t}$ evaluated at $x_{i}=q^{(2 i-1) / 2}$. Using the homogeneity of Schur functions and writing

$$
s_{\lambda^{t}}(q)=s_{\lambda^{t}}\left(1, q, q^{2}, \ldots\right)
$$

we can rewrite the right hand side of the above equation as

$$
M(q) q^{-\left(\begin{array}{c}
\lambda \\
2
\end{array}\right)} s_{\lambda^{t}}(q)
$$

Observing that

$$
\sum_{(i, j) \in \lambda}(i+j+1)=|\lambda|+\left(\begin{array}{l}
\lambda \\
2
\end{array}\right)+\left(\begin{array}{c}
\lambda^{t} \\
2
\end{array}\right)
$$

we get

$$
\sum_{n, d=0}^{\infty} p(n, d) q^{n} v^{d}=M(q)^{2} \sum_{\lambda} s_{\lambda^{t}}(q)^{2} q^{|\lambda|+\left(\begin{array}{c}
\lambda^{t} \\
2
\end{array}\right)-\left(\begin{array}{c}
\lambda \\
2
\end{array}\right)} v^{|\lambda|} .
$$


The hook polynomial formula for $s_{\lambda^{t}}(q)$ (I.3 ex 2 pg 45,[Mac95]) is

$$
s_{\lambda^{t}}(q)=q^{\left(\begin{array}{l}
\lambda \\
2
\end{array}\right)} \prod_{x \in \lambda^{t}}\left(1-q^{h(x)}\right)^{-1}
$$

from which one easily sees that

$$
s_{\lambda^{t}}(q)=q^{\left(\begin{array}{c}
\lambda \\
2
\end{array}\right)-\left(\begin{array}{c}
\lambda^{t} \\
2
\end{array}\right)} s_{\lambda}(q) .
$$

Therefore

$$
\begin{aligned}
\sum_{n, d=0}^{\infty} p(n, d) q^{n} v^{d} & =M(q)^{2} \sum_{\lambda} s_{\lambda}(q) s_{\lambda^{t}}(q) q^{|\lambda|} v^{|\lambda|} \\
& =M(q)^{2} \sum_{\lambda} s_{\lambda}\left(q, q^{2}, q^{3}, \ldots\right) s_{\lambda^{t}}\left(v, v q, v q^{2}, \ldots\right) \\
& =M(q)^{2} \prod_{i, j=1}^{\infty}\left(1+q^{i+j-1} v\right)
\end{aligned}
$$

where the last equality comes from the orthogonality of Schur functions (I.4 equation $(4.3)^{\prime}$ of $\left.[\mathrm{Mac} 95]\right)$. By rearranging this last sum and taking the $v^{d}$ term, the lemma is proved.

Remark 2.10. From the proof of the lemma we see that

$$
P_{d}(q)=q^{d} \sum_{\lambda \vdash d} s_{\lambda}(q) s_{\lambda^{t}}(q) .
$$

From Equation (3), it is immediate that $P_{d}(q)$ is a rational function in $q$. Moreover, using the formula for total hooklength (pg 11, I.1 ex 2, [Mac95]), it is easy to check that $P_{d}(q)$ is invariant under $q \mapsto 1 / q$.

\subsection{General $Y$.}

Lemma 2.11. Let $C$ be a super-rigid rational curve on the Calabi-Yau threefold $Y$. Then

$$
\widetilde{\chi}\left(\widetilde{J}_{n}(Y, d C), J_{n}(Y, d C)\right)=(-1)^{n-d} p(n, d),
$$

for all $n, d$.

Proof. First of all, by Theorem 3.2 of [La81], an analytic neighborhood of $C$ in $Y$ is isomorphic to an analytic neighborhood of $C_{0}$ in $N$. Therefore, by the analytic theory of Hilbert schemes (or Douady spaces), see [Do66], we obtain an analytic isomorphism of $\widetilde{J}_{n}(Y, d C)$ with $\widetilde{J}_{n}\left(N, d C_{0}\right)$ which extends to an isomorphism of a tubular neighborhood of $\widetilde{J}_{n}(Y, d C)$ in $I_{n}(Y,[d C])$ with a tubular neighborhood of $\widetilde{J}_{n}\left(N, d C_{0}\right)$ in $I_{n}\left(N,\left[d C_{0}\right]\right)$.

The formula for $\nu_{X}(P)$ in terms of a linking number, Proposition 4.22 of [Be05], shows that $\nu_{X}(P)$ is an invariant of the underlying analytic structure of a scheme $X$. Thus, we have

$$
\widetilde{\chi}\left(\widetilde{J}_{n}(Y, d C), I_{n}(Y,[d C])\right)=\widetilde{\chi}\left(\widetilde{J}_{n}\left(N, d C_{0}\right), I_{n}\left(N,\left[d C_{0}\right]\right)\right) .
$$

Finally, apply Corollary 2.8 . 
Lemma 2.12. Let $f: X \rightarrow Y$ be an étale morphism of separated schemes of finite type over $\mathbb{C}$. Let $Z \subset X$ be a constructible subset. Assume that the restriction of $f$ to the closed points of $Z, f: Z(\mathbb{C}) \rightarrow Y(\mathbb{C})$, is injective. Then we have

$$
\widetilde{\chi}(f(X), Y)=\tilde{\chi}(Z, X) .
$$

We remark that by Chevalley's theorem (EGA IV, Cor. 1.8.5), $f(Z)$ is constructible, so that $\tilde{\chi}(f(X), Y)$ is defined.

Proof. Without loss of generality, $Z \subset X$ is a closed subscheme and so $Z \rightarrow Y$ is unramified.

We claim that there exists a decomposition $Y=Y_{1} \sqcup \ldots \sqcup Y_{n}$ into locally closed subsets, such that, putting the reduced structure on $Y_{i}$, the induced morphism $Z_{i}=$ $Z \times_{Y} Y_{i} \rightarrow Y_{i}$ is either an isomorphism, or $Z_{i}$ is empty.

In fact, by generic flatness (EGA IV, Cor. 6.9.3), we may assume without loss of generality that $Z \rightarrow Y$ is flat, hence étale. By Zariski's Main Theorem (EGA IV, Cor. 18.12.13), we may assume that $Z \rightarrow Y$ is finite, hence finite étale. Then, by our injectivity assumption, the degree of $Z \rightarrow Y$ is 1 and so $Z \rightarrow Y$ is an isomorphism.

Once we have this decomposition of $Y$, the lemma follows from additivity of the Euler characteristic over such decompositions and the étale invariance of the canonical constructible function $\nu$.

Now we consider the case of a curve with several components. Let

$$
C_{\vec{d}}=\sum_{i=1}^{s} d_{i} C_{i}
$$

be an effective cycle, where the $C_{i}$ are pairwise disjoint super-rigid rational curves in $Y$. We assume $d_{i}>0$, for all $i=1, \ldots, s$.

For an $(s+1)$-tuple of non-negative integers $\vec{m}=\left(m_{0}, m_{1}, \ldots, m_{s}\right)$, we let $|\vec{m}|=$ $\sum_{i=0}^{s} m_{i}$. Consider, for $|\vec{m}|=n$ the open subscheme

$$
U_{\vec{m}} \subset \operatorname{Hilb}^{m_{0}}(Y) \times \prod_{i=1}^{s} J_{m_{i}}\left(Y, d_{i} C_{i}\right),
$$

consisting of subschemes $\left(Z_{0},\left(Z_{i}\right)\right)$ with pairwise disjoint support.

Lemma 2.13. Mapping $\left(Z_{0},\left(Z_{i}\right)\right)$ to $Z_{0} \cup \bigcup_{i} Z_{i}$ defines an étale morphism

$$
f: U_{\vec{m}} \longrightarrow J_{n}\left(Y, C_{\vec{d}}\right) \text {. }
$$

Proof. This is straightforward. See also Lemma 4.4 in [BF97].

Let us write $Y$ for $Y \backslash \operatorname{supp} C$ and remark that

$$
Z_{\vec{m}}=\operatorname{Hilb}^{m_{0}}(\stackrel{\circ}{Y}) \times \prod_{i>0} \widetilde{J}_{m_{i}}\left(Y, d_{i} C_{i}\right)
$$

is contained in $U_{\vec{m}}$. Moreover, the restriction $f: Z_{\vec{m}} \rightarrow J_{n}\left(Y, C_{\vec{d}}\right)$ is injective on closed points. Finally, every closed point of $J_{n}\left(Y, C_{\vec{d}}\right)$ is contained in $f\left(Z_{\vec{m}}\right)$, for a unique $\vec{m}$, such that $|\vec{m}|=n$. 
We will apply Lemma 2.12 to the diagram

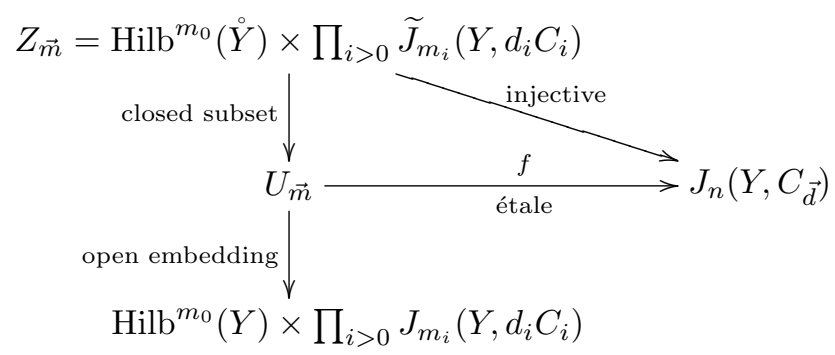

Thus, we may calculate as follows:

$$
\begin{aligned}
& \tilde{\chi}\left(J_{n}\left(Y, C_{\vec{d}}\right)\right) \\
& \quad=\sum_{|\vec{m}|=n} \tilde{\chi}\left(f\left(Z_{\vec{m}}\right), J_{n}\left(Y, C_{\vec{d}}\right)\right) \\
& =\sum_{|\vec{m}|=n} \tilde{\chi}\left(Z_{\vec{m}}, U_{\vec{m}}\right) \\
& =\sum_{|\vec{m}|=n} \tilde{\chi}\left(\operatorname{Hilb}^{m_{0}}(\stackrel{Y}{Y}) \times \prod_{i>0} \widetilde{J}_{m_{i}}\left(Y, d_{i} C_{i}\right), \operatorname{Hilb}^{m_{0}}(Y) \times \prod_{i>0} J_{m_{i}}\left(Y, d_{i} C_{i}\right)\right) \\
& =\sum_{|\vec{m}|=n} \tilde{\chi}\left(\operatorname{Hilb}^{m_{0}}(\stackrel{\circ}{Y}), \operatorname{Hilb}^{m_{0}}(Y)\right) \prod_{i>0} \tilde{\chi}\left(\widetilde{J}_{m_{i}}\left(Y, d_{i} C_{i}\right), J_{m_{i}}\left(Y, d_{i} C_{i}\right)\right) \\
& =\sum_{|\vec{m}|=n} \tilde{\chi}\left(\operatorname{Hilb}^{m_{0}}\left(\stackrel{Y}{Y}^{\prime}\right)\right) \prod_{i>0}(-1)^{m_{i}-d_{i}} p\left(m_{i}, d_{i}\right) .
\end{aligned}
$$

Now we perform the summation:

$$
\begin{aligned}
& \sum_{n=0}^{\infty} \widetilde{\chi}\left(J_{n}\left(Y, C_{\vec{d}}\right)\right) q^{n} \\
& =\sum_{n=0}^{\infty}\left(\sum_{|\vec{m}|=n} \tilde{\chi}\left(\operatorname{Hilb}^{m_{0}}(\stackrel{\circ}{Y})\right) \prod_{i=1}^{s}(-1)^{m_{i}-d_{i}} p\left(m_{i}, d_{i}\right)\right) q^{n} \\
& =\sum_{n=0}^{\infty} \sum_{|\vec{m}|=n} \tilde{\chi}\left(\operatorname{Hilb}^{m_{0}}(\stackrel{\circ}{Y})\right) q^{m_{0}} \prod_{i=1}^{s}(-1)^{d_{i}} p\left(m_{i}, d_{i}\right)(-q)^{m_{i}} \\
& =\left(\sum_{m_{0}=0}^{\infty} \tilde{\chi}\left(\operatorname{Hilb}^{m_{0}}(\stackrel{\circ}{Y})\right) q^{m_{0}}\right) \sum_{\vec{m}^{\prime}} \prod_{i=1}^{s}(-1)^{d_{i}} p\left(m_{i}, d_{i}\right)(-q)^{m_{i}} \\
& =M(-q)^{\chi(\grave{Y})} \prod_{i=1}^{s}(-1)^{d_{i}} \sum_{m_{i}=0}^{\infty} p\left(m_{i}, d_{i}\right)(-q)^{m_{i}} \\
& =M(-q)^{\chi(\stackrel{\circ}{)})} \prod_{i=1}^{s} M(-q)^{2}(-1)^{d_{i}} P_{d_{i}}(-q) \\
& =M(-q)^{\chi(Y)} M(-q)^{2 s} \prod_{i=1}^{s}(-1)^{d_{i}} P_{d_{i}}(-q)
\end{aligned}
$$




$$
=M(-q)^{\chi(Y)} \prod_{i=1}^{s}(-1)^{d_{i}} P_{d_{i}}(-q)
$$

By the main result of [Be05], Theorem 4.18, we have

$$
N_{n}\left(Y, C_{\vec{d}}\right)=\widetilde{\chi}\left(J_{n}\left(Y, C_{\vec{d}}\right)\right) .
$$

This finishes the proof of:

Theorem 2.14. The partition function for the contribution of $C_{\vec{d}}$ to the DonaldsonThomas invariants of $Y$ is given by

$$
Z\left(Y, C_{\vec{d}}\right)=\sum_{n=0}^{\infty} N_{n}\left(Y, C_{\vec{d}}\right) q^{n}=M(-q)^{\chi(Y)} \prod_{i=1}^{s}(-1)^{d_{i}} P_{d_{i}}(-q) .
$$

Corollary 2.15. Define the reduced partition function

$$
Z^{\prime}\left(Y, C_{\vec{d}}\right)=\frac{Z\left(Y, C_{\vec{d}}\right)}{Z(Y, 0)}
$$

Then we have

$$
Z^{\prime}\left(Y, C_{\vec{d}}\right)=\prod_{i=1}^{s}(-1)^{d_{i}} P_{d_{i}}(-q)
$$

a rational function in $q$, invariant under $q \mapsto 1 / q$.

Proof. Behrend and Fantechi prove $[\mathrm{BF} 05]$ that

$$
Z(Y, 0)=\sum_{n=0}^{\infty} \tilde{\chi}\left(\operatorname{Hilb}^{n} Y\right) q^{n}=M(-q)^{\chi(Y)}
$$

the formula for $Z^{\prime}\left(Y, C_{\vec{d}}\right)$ then follows immediately from Theorem 2.14. For the proof that $Z^{\prime}\left(Y, C_{\vec{d}}\right)$ is a rational function invariant under $q \mapsto 1 / q$, see Remark 2.10.

\section{The super-rigid GW/DT correspondence.}

3.1. The usual GW/DT correspondence. We can formulate the The GromovWitten/Donaldson-Thomas correspondence of [MNOP] as follows.

Let $Y$ be a Calabi-Yau threefold and let

$$
Z_{D T}(Y, \beta)=\sum_{n \in \mathbb{Z}} N_{n}(Y, \beta) q^{n}
$$

be the partition function for the degree $\beta$ Donaldson-Thomas invariants. Let

$$
Z_{D T}^{\prime}(Y, \beta)=\frac{Z_{D T}(Y, \beta)}{Z_{D T}(Y, 0)}
$$

be the reduced partition function.

In Gromov-Witten theory, the reduced partition function for the degree $\beta$ GromovWitten invariants, $Z_{G W}^{\prime}(Y, \beta)$, is given by the coefficients of the exponential of the $\beta \neq 0$ part of the potential function:

$$
1+\sum_{\beta \neq 0} Z_{G W}^{\prime}(Y, \beta) v^{\beta}=\exp \left(\sum_{\beta \neq 0} N_{g}^{G W}(Y, \beta) u^{2 g-2} v^{\beta}\right) .
$$


Here

$$
N_{g}^{G W}(Y, \beta)=\operatorname{deg}\left[\bar{M}_{g}(Y, \beta)\right]^{\mathrm{vir}}
$$

is the genus $g$, degree $\beta$ Gromov-Witten invariant of $Y$.

The conjectural GW/DT correspondence states that

(i) The degree 0 partition function in Donaldson-Thomas theory is given by

$$
Z_{D T}(Y, 0)=M(-q)^{\chi(Y)}
$$

(ii) $Z_{D T}^{\prime}(Y, \beta)$ is a rational function in $q$, invariant under $q \mapsto 1 / q$, and

(iii) the equality

$$
Z_{G W}^{\prime}(Y, \beta)=Z_{D T}^{\prime}(Y, \beta)
$$

holds under the change of variables $q=-e^{i u}$.

Part (i) is proved for all $Y$ in [BF05].

3.2. The super-rigid GW/DT correspondence. In an entirely parallel manner, we can formulate the GW/DT correspondence for $N_{n}\left(Y, C_{\vec{d}}\right)$, the contribution from a collection of super-rigid rational curves $C_{\vec{d}}=\sum_{i} d_{i} C_{i}$.

Just as in Donaldson-Thomas theory, there is an open component of the moduli space of stable maps

$$
\bar{M}_{g}\left(Y, C_{\vec{d}}\right) \subset \bar{M}_{g}(Y, \beta)
$$

parameterizing maps whose image lies in the support of $C_{\vec{d}}$. There are corresponding invariants given by the degree of the virtual class:

$$
N_{g}^{G W}\left(Y, C_{\vec{d}}\right)=\operatorname{deg}\left[\bar{M}_{g}\left(Y, C_{\vec{d}}\right)\right]^{\mathrm{vir}}
$$

We define $Z_{G W}^{\prime}\left(Y, C_{\vec{d}}\right)$ by replacing $N_{g}^{G W}(Y, \beta)$ on the right side of formula (4) by $N_{g}^{G W}\left(Y, C_{\vec{d}}\right)$.

Then we can formulate our results as follows.

Theorem 3.1. The $G W / D T$ correspondence holds for the contributions from superrigid rational curves. Namely, let $C_{\vec{d}}=d_{1} C_{1}+\cdots+d_{s} C_{s}$ be a cycle supported on pairwise disjoint super-rigid rational curves $C_{i}$ in a Calabi-Yau threefold $Y$, and let $Z_{D T}^{\prime}\left(Y, C_{\vec{d}}\right)$ and $Z_{G W}^{\prime}\left(Y, C_{\vec{d}}\right)$ be defined as above. Then

(ii) $Z_{D T}^{\prime}\left(Y, C_{\vec{d}}\right)$ is a rational function of $q$, invariant under $q \mapsto 1 / q$, and

(iii) the equality

$$
Z_{D T}^{\prime}\left(Y, C_{\vec{d}}\right)=Z_{G W}^{\prime}\left(Y, C_{\vec{d}}\right)
$$

holds under the change of variables $q=-e^{i u}$.

Proof. For (ii), see Corollary 2.15. To prove (iii), we reproduce a calculation well known to the experts (e.g. [Ka06]).

By the famous multiple cover formula of Faber-Pandharipande [FP00] (see also [Pa99]),

$$
N_{g}^{G W}\left(Y, C_{\vec{d}}\right)=\sum_{i=1}^{s} c\left(g, d_{i}\right)
$$

where $c(g, d)$ is given by

$$
\sum_{g \geq 0} c(g, d) u^{2 g-2}=\frac{1}{d}\left(\sin \left(\frac{d u}{2}\right)\right)^{-2} .
$$


We compute $Z_{G W}^{\prime}\left(Y, C_{\vec{d}}\right)$ and make the substitution $q=-e^{i u}$ :

$$
\begin{aligned}
1+\sum_{\left(d_{1}, \ldots, d_{s}\right) \neq 0}^{\infty} Z_{G W}^{\prime}\left(Y, C_{\vec{d}}\right) v_{1}^{d_{1}} \cdots v_{s}^{d_{s}} \\
=\exp \left(\sum_{j=1}^{s} \sum_{d_{j}=1}^{\infty} \sum_{g=0}^{\infty} c\left(g, d_{j}\right) u^{2 g-2} v_{j}^{d_{j}}\right) \\
=\prod_{j=1}^{s} \exp \left(\sum_{d_{j}=1}^{\infty} \frac{v_{j}^{d_{j}}}{d_{j}}\left(2 \sin \frac{d_{j} u}{2}\right)^{-2}\right) \\
=\prod_{j=1}^{s} \exp \left(\sum_{d_{j}=1}^{\infty} \frac{v_{j}^{d_{j}}}{d_{j}} \frac{-e^{i d_{j} u}}{\left(1-e^{i d_{j} u}\right)^{2}}\right) \\
=\prod_{j=1}^{s} \exp \left(\sum_{d_{j}=1}^{\infty} \sum_{m_{j}=1}^{\infty} \frac{-m_{j}}{d_{j}} e^{i d_{j} m_{j} u} v_{j}^{d_{j}}\right) \\
=\prod_{j=1}^{s} \exp \left(\sum_{m_{j}=1}^{\infty} m_{j} \log \left(1-v_{j} e^{i m_{j} u}\right)\right) \\
=\prod_{j=1}^{s} \prod_{m_{j}=1}^{\infty}\left(1-(-q)^{m_{j}} v_{j}\right)^{m_{j}} \\
=\prod_{j=1}^{s} \sum_{d_{j}=0}^{\infty} P_{d_{j}}(-q)\left(-v_{j}\right)^{d_{j}} \\
=\sum_{\left(d_{1}, \ldots, d_{s}\right)}^{s} \prod_{j=1}^{s}(-1)^{d_{j}} P_{d_{j}}(-q) v_{j}^{d_{j}} .
\end{aligned}
$$

Therefore,

$$
Z_{G W}^{\prime}\left(Y, C_{\vec{d}}\right)=\prod_{j=1}^{s}(-1)^{d_{j}} P_{d_{j}}(-q)
$$

and so by comparing with Corollary 2.15 the theorem is proved.

The following corollary is immediate.

Corollary 3.2. Let $Y$ be a Calabi-Yau threefold and let $\beta \in H_{2}(Y, \mathbb{Z})$ be a curve class such that all cycle representatives of $\beta$ are supported on a collection of pairwise disjoint, super-rigid rational curves. Then the degree $\beta G W / D T$ correspondence holds:

$$
Z_{D T}^{\prime}(Y, \beta)=Z_{G W}^{\prime}(Y, \beta) .
$$

For example, we have:

Corollary 3.3. Let $Y \subset \mathbb{P}^{4}$ be a quintic threefold, and let $L$ be the class of the line. Then for $\beta$ equal to $L$ or $2 L$, the $G W / D T$ correspondence holds. 
Proof. By deformation invariance of both Donaldson-Thomas and Gromov-Witten invariants, it suffices to let $Y$ be a generic quintic threefold. It is well known that there are exactly 2875 pairwise disjoint lines on $Y$ and they are all super-rigid. The conics on $Y$ are all planar and hence rational, and it is known that there are exactly 609250 pairwise disjoint conics and they are super-rigid as well. For these facts and more, see [Ka86].

Note that we cannot prove the GW/DT conjecture by this method for the quintic in degree three (and higher) due to the presence of elliptic curves in degree three.

Explicit formulas for the reduced Donaldson-Thomas partition function of a generic quintic threefold $Y$ in degrees one and two are given below:

$$
\begin{aligned}
Z_{D T}^{\prime}(Y, L) & =2875 \frac{q}{(1-q)^{2}} \\
Z_{D T}^{\prime}(Y, 2 L) & =609250 \frac{q}{(1-q)^{2}} \cdot 2875 \cdot \frac{-2 q^{3}}{(1+q)^{4}(1-q)^{2}} \\
& =-3503187500 \frac{1}{\left(q-q^{-1}\right)^{4}}
\end{aligned}
$$

\section{References}

[Be05] K. Behrend. Donaldson-Thomas type invariants via microlocal geometry. ArXiv: math.AG/0507523. To appear in Ann. Math.

[BF05] K. Behrend and B. Fantechi. Symmetric obstruction theories and Hilbert schemes of points on threefolds. ArXiv: math.AG/0512556.

[BF97] K. Behrend and B. Fantechi. The intrinsic normal cone. Invent. Math., 128(1):45-88, 1997.

[BP01] J. Bryan and R. Pandharipande. BPS states of curves in Calabi-Yau 3-folds. Geom. Topol., 5:287-318 (electronic), 2001.

[Do66] A. Douady. Le problème des modules pour les sous-espaces analytiques compacts d'un espace analytique donné. Ann. Inst. Fourier (Grenoble), 16(fasc. 1):1-95, 1966.

[FP00] C. Faber and R. Pandharipande. Hodge integrals and Gromov-Witten theory. Invent. Math., 139(1):173-199, 2000.

[Ka86] S. Katz. On the finiteness of rational curves on quintic threefolds. Compositio Math., 60(2):151-162, 1986.

[Ka06] S. Katz. Gromov-Witten, Gopakumar-Vafa, and Donaldson-Thomas invariants of CalabiYau threefolds. In Snowbird lectures on string geometry, volume 401 of Contemp. Math., pages 43-52. Amer. Math. Soc., Providence, RI, 2006.

[Ko96] J. Kollár. Rational curves on algebraic varieties, volume 32 of Ergebnisse der Mathematik und ihrer Grenzgebiete. 3. Folge. Springer-Verlag, Berlin, 1996.

[La81] H. B. Laufer. On $\mathbf{C} P^{1}$ as an exceptional set. In Recent developments in several complex variables, volume 100 of Ann. of Math. Stud., pages 261-275. Princeton, N.J., 1981.

[Li06] J. Li. Zero dimensional Donaldson-Thomas invariants of threefolds. Geom. Topol., 10:21172171 (electronic), 2006.

[LP06] M. Levine and R. Pandharipande. Algebraic cobordism revisited. arXiv:math.AG/0605196.

[Mac95] I. G. Macdonald. Symmetric functions and Hall polynomials. Oxford Mathematical Monographs. New York, second edition, 1995.

[MNOP] D. Maulik, N. Nekrasov, A. Okounkov, and R. Pandharipande. Gromov-Witten theory and Donaldson-Thomas theory, I. Compositio Math.. 142(5):1263-1285, 2006.

[ORV] A. Okounkov, N. Reshetikhin, and C. Vafa. Quantum Calabi-Yau and classical crystals. In The unity of mathematics, volume 244 of Progr. Math., pages 597-618. Birkhäuser Boston, Boston, MA, 2006.

[Pa99] R. Pandharipande. Hodge integrals and degenerate contributions. Comm. Math. Phys., 208(2):489-506, 1999. 
[Th00] R. P. Thomas. A holomorphic Casson invariant for Calabi-Yau 3-folds, and bundles on K3 fibrations. J. Differential Geom., 54(2):367-438, 2000.

Department of Mathematics, The University of British Columbia, 1984 Mathematics Road, Vancouver, B.C., Canada V6T $1 Z 2$

E-mail address: behrend@math.ubc.ca

Department of Mathematics, The University of British Columbia, 1984 Mathematics

Road, Vancouver, B.C., Canada V6T $1 Z 2$

E-mail address: jbryan@math.ubc.ca 